\title{
Evaluating the Economic Burden of Relapses in Neuromyelitis Optica Spectrum Disorder: A Real- World Analysis Using German Claims Data
}

\author{
Rachel K. Knapp (D) · Fränce Hardtstock • Thomas Wilke • \\ Ulf Maywald · Barthold Deiters · Sophie Schneider · Julie Mouchet
}

Received: October 11, 2021 / Accepted: December 1, 2021 / Published online: December 23, 2021

(C) The Author(s) 2021

\begin{abstract}
Introduction: Neuromyelitis optica spectrum disorder (NMOSD) is a rare autoimmune disease of the central nervous system which causes recurrent relapses, resulting in blindness, paralysis, and spinal cord damage. This study sought to explore the real-world burden, treatment, and cost of NMOSD in Germany using claims data.
\end{abstract}

Methods: Our study consisted of a retrospective analysis of two anonymized health insurance datasets covering around 9 million patients in

Supplementary Information The online version contains supplementary material available at https:// doi.org/10.1007/s40120-021-00311-x.

R. K. Knapp $(\bowtie) \cdot$ F. Hardtstock

Cytel Inc., Potsdamer Str. 58, 10785 Berlin, Germany

e-mail: rachel.knapp@ingress-health.com

T. Wilke

IPAM E.V., Alter Holzhafen 19, 23966 Wismar,

Germany

U. Maywald

AOK PLUS, Sternplatz 7, 01067 Dresden, Germany

B. Deiters

GWQ ServicePlus AG, Tersteegenstraße 28, 40474

Düsseldorf, Germany

S. Schneider $\cdot$ J. Mouchet

F. Hoffmann-La Roche Ltd, Grenzacherstrasse 124,

4070 Basel, Switzerland
Germany from $01 / 01 / 2013$ to $31 / 12 / 2019$. NMOSD patients were identified using inpatient and outpatient International Classification of Diseases, Tenth Revision (ICD-10) diagnoses of neuromyelitis optica (NMO; G36.0) and relevant symptom codes. Active periods of disease were identified based on relapse events (including hospitalizations and acute treatment); healthcare resource utilization (HCRU) and direct costs were allocated to active and inactive periods based on treatment dates. Propensity score matching was used to compare HCRU and cost outcomes among patients with and without NMOSD.

Results: Overall, 130 patients were identified as having NMOSD (mean age: 46.84 years; 58\% female). NMOSD patients recorded 16.52 active and 348.48 inactive days per patient year (PPY). HCRU and associated costs were approximately tenfold higher during active periods than during inactive periods, with the largest share of the cost difference driven by hospitalizations (€6424.09/€259.10 per active/inactive month) and outpatient drug prescriptions (€412.83/ $€ 271.58)$. Direct healthcare costs incurred by patients with NMOSD (€12,913.28 PPY) were approximately threefold higher than those incurred by patients without NMOSD ( $€ 4667.66$ PPY). Costs of hospitalization (€6448.32/ $€ 1937.64 \mathrm{PPY})$ and outpatient prescriptions (€3335.67/€1037.64 PPY) contributed most strongly to the difference. 
Conclusion: Patients with NMOSD consume substantial healthcare resources and incur heavy costs during active disease phases. This study captured direct measurable healthcare costs and likely underestimates the real societal/ emotional burden on patients and their families. Nevertheless, prevention of acute relapses represents one compelling strategy to minimize the economic burden of NMOSD in Germany.

Keywords: Claims data; Direct healthcare costs; Germany; Healthcare resource use; Neuromyelitis optica spectrum disorder; Propensity score matching; Real-world evidence; Relapse

\section{Key Summary Points}

\section{Why carry out this study?}

To expand upon a growing body of claims data research from the United States and East Asia, this study sought to assess the healthcare resource and cost burden of neuromyelitis optica spectrum disorder (NMOSD) in Germany during periods of active and inactive disease, as well as amongst patients with NMOSD and a propensity score-matched comparator group.

We hypothesized that the costs of care would be highest during periods of active disease and that NMOSD patients would incur higher costs than the comparator group of individuals without NMOSD.

\section{What was learned from the study?}

Annualized direct healthcare spending was found to be almost three times as high in NMOSD patients as compared to the propensity score-matched cohort of patients without NMOSD, and the highest degree of cost accumulation occurred within hospital settings during periods of active relapse.
Our findings suggest that heavy direct healthcare costs incurred by patients with NMOSD are strongly linked to periods of acute relapse/active disease, and that the magnitude of the cost difference in Germany is tenfold higher during active periods than during inactive periods.

Our results reaffirm that hospitalization and outpatient prescriptions constitute the primary cost drivers when treating active NMOSD.

\section{INTRODUCTION}

Neuromyelitis optica spectrum disorder (NMOSD), also known as Devic's disease and neuromyelitis optica (NMO), is a rare autoimmune condition of the central nervous system (CNS), primarily characterized by the inflammation of the optic nerve [optic neuritis $(\mathrm{ON})$ ] and spinal cord [longitudinal extensive transverse myelitis (LETM)] [1]. When left untreated, patients with NMOSD may suffer from acute relapses, resulting in blindness, paralysis, and death. Various studies have suggested that NMOSD is often misdiagnosed as multiple sclerosis (MS) in clinical practice, given its low prevalence (approximately $0.5-4$ cases $/ 100,000$ ) $[2,3]$ and its similar profile as an autoimmune disease which involves the loss of visual acuity as well as weakness, numbness and pain associated with the demyelination of the spinal cord $[4,5]$. In recent years, serological testing for highly specific autoantibodies targeting the aquaporin-4 water channel (AQP4-IgG) has been used to detect NMOSD in clinical settings, with an average sensitivity of over $75 \%$ when using cell-based serum assays [6].

NMOSD is frequently characterized by recurrent and debilitating relapses, which involve the onset of new or worsening symptoms and may result in partial or bilateral blindness, weakness, paralysis, loss of sensation, digestive dysfunction, and spinal cord damage [7]. While some symptoms of NMOSD attacks and recurrent relapses are reversible, active 
phases have been found to have a profound, negative impact on quality of life of NMOSD patients and instances of permanent neurological disability are common $[8,9]$. Attacks and relapses have been found to occur almost twice as frequently in NMOSD as in MS, and are associated with more severe neurological impairments, as well as lower rates of full recovery $[10,11]$.

Generally, treatment of NMOSD comprises three main types: (1) acute therapy, (2) maintenance therapy, and (3) treatment of symptoms $[12,13]$. The objective of acute treatment is to subdue inflammatory attacks, minimize damage to the CNS, and improve long-term neurological function $[12,13]$. Acute treatment of NMOSD generally consists of a 5-day highdose regimen of corticosteroids (often intravenous methylprednisolone) followed by a $2-8$ week-long oral steroid taper $[12,13]$. In some cases, plasma exchange (PLEX) and/or intravenous immunoglobulin (IVIG) are used in patients with NMOSD to regain neurological function after an attack/relapse. Following a patient's initial diagnosis, long-term immunosuppressive therapy is used as a maintenance therapy to reduce the risk of relapse. Maintenance treatment regimens for NMOSD may vary; however, common off-label therapies include oral corticosteroids, azathioprine, rituximab, and mycophenolate, among other immunosuppressant drugs like mitoxantrone, methotrexate, and tocilizumab [12-14]. Since 2019 , three new therapies-eculizumab, inebilizumab, and satralizumab-have been approved. Among these, only eculizumab was approved in Germany at the time of this analysis. Common medications used in the treatment of NMOSD-related pain include antiepileptics, anti-spasmodic drugs, anti-depressants, and analgesics [12].

A recent study concluded that progressive impairment in patients with NMOSD is singularly linked to clinical relapses and that no depreciation in the Expanded Disability Status Scale (EDSS) score was detectable during inactive disease periods [15]. These findings, among others, have placed further emphasis on the prevention of relapse as a means of optimizing clinical outcomes for patients with NMOSD. In alignment with a small but growing body of claims research from the United States (USA) and East Asia [16-20], this study seeks to explore the real-world burden, treatment, and direct measurable costs of NMOSD in Germany using claims data, and to explore the differential effect of active and inactive disease phases on the costs of treating NMOSD patients. Study objectives also included a comparison of healthcare resource utilization (HCRU) and costs incurred by patients with NMOSD and a matched cohort of comparators without NMOSD.

\section{METHODS}

\section{Patient Identification and Active Period Assignment}

This study consisted of a non-interventional, retrospective analysis of anonymized health insurance claims datasets provided by AOK PLUS and GWQ ServicePlus during the period from $01 / 01 / 2013$ to $31 / 12 / 2019$. Taken together, the data from both providers covered a total sickness fund population of around 9 million patients in Germany (3.2 and 5.7 million patients, respectively). Patients with NMOSD (as well as a matched comparison group of patients without NMOSD) were identified via an algorithm using inpatient and outpatient International Classification of Diseases, Tenth Revision, German modification (ICD-10-GM) diagnostic codes.

Patients with NMOSD were identified if they received at least (1) one inpatient code for neuromyelitis optica (G36.0), or (2) two confirmed outpatient diagnoses of NMO, or (3) a confirmed outpatient diagnosis of NMO by a relevant specialist $^{1}$ and at least one inpatient or confirmed outpatient diagnosis of optic neuritis (H46), or transverse myelitis (TM) (G37.3) by a relevant specialist during the inclusion period (01/01/2014-31/12/2018). The first observed NMO, TM, or ON code within the inclusion

\footnotetext{
1 Within the context of this study, the term relevant specialist refers to the following specialties: neurology, neuroradiology, ophthalmology, and rheumatology.
} 
period was set as the index date and all patients included in the analysis were required to be continuously insured by their respective sickness fund for a minimum period of 2 years (with at least 12 months of baseline and follow-up observation). The only exception to the minimum follow-up condition was early death. Generally, patients were excluded based on subsequent inpatient and outpatient specialist codes for multiple sclerosis (G35) and sarcoidosis (D86); however, a systematic check of these patients' diagnostic sequence was performed to account for the strong likelihood of potential MS misdiagnosis, especially in the early phase of NMOSD diagnosis. Consequently, patients who only received a few subsequent outpatient MS codes close to the index date (with a much higher proportion of subsequent inpatient and outpatient NMO codes) were not excluded. In addition to the base cohort of NMOSD patients, relevant analyses were also performed for a sensitivity cohort of NMOSD patients, who were only included if outpatient diagnoses of NMO (G36.0), included in the initial eligibility criteria, were made by relevant specialists.

Patient characteristics were measured during the 1-year baseline period, while treatment was measured during the 1-year post-index period. Active disease periods were identified during the patient-individual follow-up period [from the index diagnosis until censorship, (i.e., death, end of insurance, or 31/12/2019)] based on events associated with acute attacks and/or relapses.

Within the German system, patients may enter the hospital in one of three ways: (1) via an emergency room (ER) visit with a subsequent admission by an ER doctor, (2) at the suggestion of an outpatient general practitioner (GP) or specialist, who recommends hospitalization in order to guarantee further monitoring of the patient's condition, or (3) in the event of a planned procedure/surgery. During inpatient stays in Germany, patients receive various diagnoses, recorded via ICD-10 codes. The most important of these is the main diagnosis, which provides the basis for billing/reimbursement, and generally encompasses the underlying reason for the hospitalization. In addition to this main code, a range of other primary and secondary codes are also logged, which may cover a diverse array of symptoms, infections, and conditions that were detected and treated during the inpatient stay. In our study, attack/ relapse events were defined as scenarios involving (1) hospitalization with a main diagnosis of NMO, TM or ON, or (2) treatment with acute therapies (based on outpatient codes for intravenous corticosteroids, ${ }^{2}$ or either inpatient or outpatient treatment with IVIG or PLEX). This derives from the assumption that an acute relapse, defined by the appearance of new or worsening NMOSD-related symptoms, would require immediate treatment and qualify as the main diagnosis associated with an inpatient stay. Ultimately, the start date of a relapse event was defined as either the date of the hospital admission or the date of the acute treatment code.

In line with findings from a case control study [10] and previous claims data research [17], each 30-day period following a relapse event was defined as an active period, in order to account for the prescribed therapy course for acute attacks (normally the administration of intravenous methylprednisolone for 5 days followed by oral steroids), which warrants treatment of the patient over several days or weeks. Clusters of acute treatment codes within 30 days of one another did not result in the extension of the active period; however, all hospitalizations with a main NMO, TM, or ON code were considered active, even if the duration was longer than 30 days. All periods not characterized as active were defined as inactive. HCRU and costs were allocated to active and inactive periods based on respective treatment dates and reported days per patient year (PPY) from the index diagnosis until censorship. HCRU variables covered inpatient care (hospitalization and rehabilitation stays), outpatient visits (to GPs and specialists), and sick leave periods. Cost analyses included expenses related to hospitalizations, rehabilitations, and outpatient prescriptions, as well as medical aids (e.g., mobility devices, hearing aids) and therapeutic

\footnotetext{
2 Intravenous corticosteroids were observed only in an outpatient treatment setting based on Anatomical Therapeutic Chemical (ATC) codes, since no data on inpatient use of corticosteroids was available.
} 
remedies (e.g., osteopathy, acupuncture). Outof-pocket expenses were omitted from this analysis since they are not recorded in the claims data; however, these are assumed to be negligible within the German treatment setting. In cases where an inpatient hospitalization, rehabilitation stay or sick leave period spanned both active and inactive periods, costs associated with each were allocated equitably across all days and proportionately assigned to active and inactive periods. The admission date or the first sick leave day was used to characterize hospitalization, rehabilitation, and sick leave periods in aggregate; however, the number of days allocated to active and inactive periods was determined based on the distribution of days. Figure 1 summarizes the methods used to assign days to active and inactive NMOSD phases, based on hospitalizations with a main $\mathrm{NMO} /$ $\mathrm{TM} / \mathrm{ON}$ diagnosis as well as acute treatment codes.

\section{Identification of Unmatched and Matched Cohorts of Patients Without NMOSD}

In order to compare HCRU and costs incurred by NMOSD patients with those from a matched cohort of patients without NMOSD, a second base cohort of patients was identified. Patients without NMOSD were required to satisfy

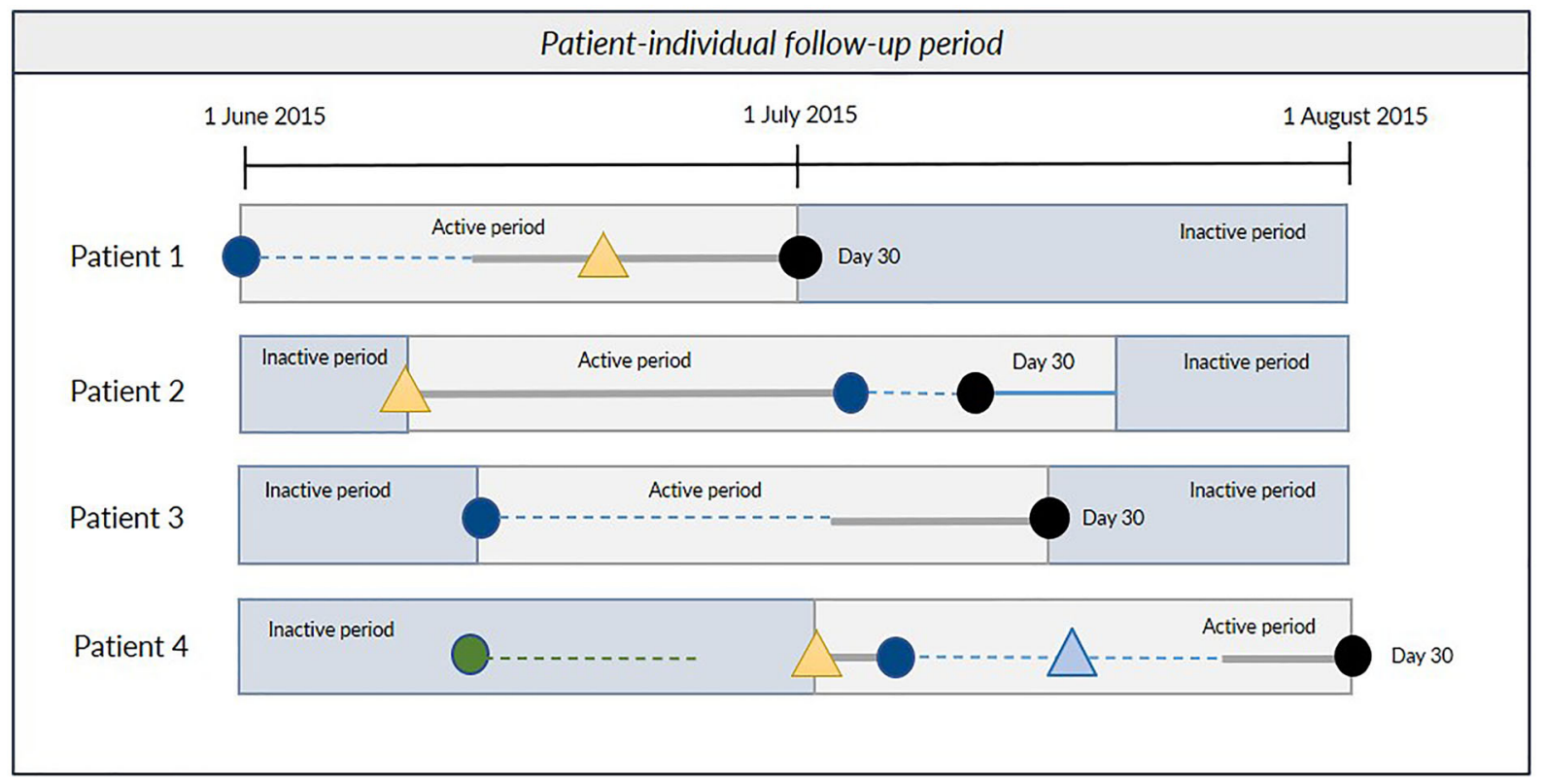

\begin{abstract}
Hospital admission with NMO/TM/ON as the main diagnosis

Hospitalization with NMO/TM/ON as main diagnosis during an active period

Hospitalization lasted longer than 30 days, resulting in an extension of the active period until the patient's discharge from the hospital

Hospital admission with alternate (non-NMO-related) main diagnosis
\end{abstract}

Fig. 1 Delineation of active and inactive disease periods based on relapse events. Summary of the methodology used to assign observational days to active and inactive NMOSD periods based on hospitalizations with a main diagnosis of NMO, TM, or ON, as well as acute treatment

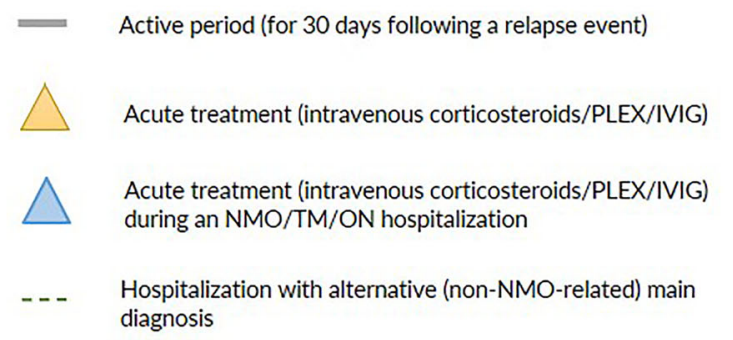

codes. IVIG intravenous immunoglobulin, NMO neuromyelitis optica, $O N$ optic neuritis, PLEX plasma exchange, $T M$ transverse myelitis 
continuous insurance criteria for the entire study period and to possess no record of inpatient or outpatient codes for $\mathrm{NMO}, \mathrm{TM}, \mathrm{ON}$, sarcoidosis, or MS from 01/01/2013 to 31/12/ 2019. The latter two codes were excluded to ensure that no NMOSD patients with current misdiagnoses of sarcoidosis and MS would be included in the non-NMOSD group. To align the observational periods between both cohorts, unmatched non-NMOSD individuals were assigned a random index date that fell between the first and last observed index date from the NMOSD patient population.

A propensity score matching (PSM) method was used to compare HCRU and cost across both groups. The PSM used a nearest-neighbor matching technique with a maximum caliper of 0.001. Patients from both cohorts were matched with those who had the same probabilistic likelihood of having NMOSD (based on a multivariate logistic regression using covariates measured during the baseline period). Covariates in the logistic regression model used to assign propensity scores included sociodemographic characteristics, previous health resource use (e.g., hospitalizations, procedures, prescriptions, outpatient visits) and comorbidities. A sensitivity analysis of different one-to-many approaches was conducted to identify the best method for matching patients with the goal of minimizing the percentage bias between matched cohorts based on observable covariates (age, sex, and comorbidities, as well as HCRU during the baseline period). Propensity scores and matched controls were assigned to patients within each dataset separately, while HCRU and costs were reported on an aggregate level for patients with and without NMOSD. Lastly, given the well-known skewness of per-patient healthcare costs and the potential impact of high-cost cases on the robustness of findings, a sensitivity cost analysis was performed in which the top and bottom $2.5 \%$ of patients in terms of total healthcare costs (related to outpatient prescriptions, outpatient care and hospitalization costs only) were excluded.

\section{Statistical Analyses}

A descriptive analysis of baseline characteristics and treatment was performed for all NMOSD patients. Treatment codes can be found in the Supplementary Material. Categorical variables were analyzed using absolute and relative frequencies while continuous variables were reported using sample statistics [including mean, standard deviation (SD), minimum, median, and maximum values]. HCRU and cost variables were measured over a minimum period of 365 days (except in cases of early death) and reported PPY, to account for the variable length of follow-up periods. Rate ratios were computed for each of the reported HCRU and cost variables, and significance was assessed based on two-sided $p$-values. All statistical analyses were performed in STATA (StataCorp. 2019. Stata Statistical Software: Release 16. College Station, TX, USA: StataCorp LLC), Microsoft SQL Server 2019, or Microsoft Excel 2019 (Microsoft Corporation, Redmond, WA, USA). This study involved the use of two anonymized health insurance claims datasets provided by AOK PLUS and GWQ ServicePLUS under formal agreement and legal basis of §75, Tenth Book of the Social Code (SGB X). No ethical approval from an institutional review board was required to implement this study, as a result of the anonymization of all patient data provided by the sickness funds.

\section{RESULTS}

\section{Patient Characteristics and Treatment of NMOSD}

Table 1 provides an overview of the baseline characteristics of NMOSD patients, as well as those included in the sensitivity cohort. A total of 130 patients were identified as having NMOSD, while 98 patients satisfied the more rigorous eligibility criteria for the sensitivity cohort. Among the base cohort, the mean age was 46.84 years, with a range of 3-89 years. Approximately $58 \%$ of the NMOSD patients were female, and five adolescent patients were included in the analysis. The average age of 
Table 1 Baseline characteristics

\section{Patient characteristics}

\section{Total patients}

$N=X$

Sex (measured at index)

Male-N (\%)

Female-N $(\%)$

Age (measured at index)

Mean (SD)

Median (range)

Charlson Comorbidity Index score (measured during the baseline period)

Median (range)

Mean (SD)

Magnetic resonance imaging (measured during the baseline period)

Either or both MRIs-N (\%)

Spinal cord MRI only-N (\%)

Brain MRI only-N (\%)

Spinal cord + brain MRI-N (\%)

Top-10 co-diagnoses (measured during the baseline period)

Dorsalgia (M54) $-N(\%)$

Essential (primary) hypertension (I10) $-N(\%)$

Disorders of lipoprotein metabolism and other lipidemias (E78) $-N(\%)$

Other acute disseminated demyelination $(\mathrm{G} 36)-N(\%)$

Disorders of refraction and accommodation (H52) $-N(\%)$

Visual disturbances (H53) $-N(\%)$

Disturbances of skin sensation (R20) $-N(\%)$

Depressive episode (F32) $-N(\%)$

Somatoform disorders (F45) $-N(\%)$

Other dorsopathies, not elsewhere classified (M53) $-N(\%)$

Length of follow-up period (in years)

Mean (SD)

$3.99(1.76)$

$10(8 \%)$

0.019

$50(38 \%)$

$44(45 \%)$

$38(29 \%)$

$31(32 \%)$

$34(26 \%)$

$30(31 \%)$

$30(23 \%)$

$26(27 \%)$

$30(23 \%)$

$25(26 \%)$

$24(18 \%)$

$24(24 \%)$

$23(18 \%)$

$23(23 \%)$

$22(17 \%)$

$21(21 \%)$

$21(16 \%)$

$20(20 \%)$

$21(16 \%)$

$17(17 \%)$

Death (measured during the follow-up period)

$N(\%)$

Death rate (per year) 
patients included in the sensitivity analysis (46.64 years) was comparable to the base cohort; however, the percentage of females was noticeably higher, at $64 \%$. During the baseline period, $42 \%$ of patients $(50 \%$ in the sensitivity cohort) received a code for diagnostic imaging in the form of brain magnetic resonance imaging (MRI) or spinal cord MRI. The average length of the follow-up period was 3.99 years, with a range of 0.12-6.00 years. Overall, 10 patients died during the follow-up period, yielding a death rate of 0.019 per year. The most common co-diagnoses (measured via three-digit ICD-10 codes during the baseline period) are also included in Table 1.

During one year of observation, 23\% of patients received some form of acute treatment with intravenous corticosteroids (11\%), plasma exchange $(13 \%)$, or intravenous immunoglobulin (3\%), and $42 \%$ of patients received maintenance therapies including oral corticosteroids (21\%), immunosuppressive therapies (12\%), and B-cell-targeting therapies (18\%). The top maintenance therapy agents were rituximab $(18 \%)$, prednisolone $(17 \%)$, and azathioprine (10\%). In addition to acute and maintenance therapies, a majority of NMOSD patients (63\%) received medicines used for pain relief (as defined in the Supplementary Material), including antiepileptic drugs (32\%), anti-inflammatory and antirheumatic drugs (32\%), analgesics (25\%), and psychoanaleptics (25\%).

\section{Characteristics of NMOSD Relapses}

Approximately 53\% in the base cohort of NMOSD patients suffered from at least one documented relapse during the follow-up period, though the percentage of patients with a relapse event was much higher in the sensitivity cohort (66\%). Based on these events, NMOSD patients recorded 16.52 active disease days PPY, as compared to 348.48 inactive days PPY, with a corresponding ratio of 0.05 active/inactive days. Ultimately, the number of active days was higher in the sensitivity cohort (21.92 days PPY). An annualized relapse rate (ARR) was calculated by dividing the total number of active periods across all patients by the total observational time. This yielded respective ARRs of 0.52 and 0.66 for the base and sensitivity cohorts.

\section{HCRU and Cost During Active and Inactive Periods}

Table 2 below compares differences in HCRU between NMOSD patients during active and inactive periods. Across all domains, HCRU was higher during active periods than during inactive periods. For each active year of observation, NMOSD patients in the base cohort recorded 13.17 all-cause outpatient visits to any doctor, as compared to 11.90 visits per each inactive year of observation. Similar trends were observed for all-cause visits to relevant specialists (2.77 PPY/2.24 PPY), visits to any doctor with a corresponding diagnosis of NMO, TM, or ON (6.18 PPY/4.66 PPY), and visits to a relevant specialist with a corresponding diagnosis of NMO, TM or ON (1.83 PPY/0.91 PPY).

Patients recorded 9.29 all-cause hospitalizations and 88.95 hospitalization days during each active year of observation, as compared to 0.52 all-cause hospitalizations and 6.11 hospitalization days during inactive years. This result is mainly driven by NMOSD-related stays; however, the difference across active and inactive periods is also present for non-NMOSD-related stays (e.g., those not resulting in a diagnosis of NMO, TM or $\mathrm{ON}$ ), with 0.47 active and 0.40 inactive hospitalizations PPY, and 8.06 active and 5.04 inactive hospitalization days PPY. Patients with active NMOSD recorded 3.81 times as many sick leave days as they did during inactive periods, and 9.96 times as many sick leave days in which the corresponding diagnosis was related to NMOSD.

The total healthcare costs incurred per patient month (PPM) of active observation was $€ 7159.08$, as opposed to $€ 714.17$ PPM of inactive observation. This corresponds to a tenfold difference in cost across active and inactive periods, with the largest share of the cost difference driven by inpatient hospitalizations (€6424.09/€259.10 per active/inactive month) and outpatient prescriptions (€412.83/€271.58). Table 3 provides an overview of direct costs 
Table 2 Comparison of HCRU during active and inactive disease phases

\begin{tabular}{|c|c|c|c|c|}
\hline \multirow{2}{*}{$\begin{array}{l}\text { Healthcare resource utilization } \\
\text { (measured during the follow-up period) }\end{array}$} & \multicolumn{2}{|l|}{ Active } & \multicolumn{2}{|l|}{ Inactive } \\
\hline & Base cohort & Sensitivity cohort & Base cohort & Sensitivity cohort \\
\hline \multicolumn{5}{|l|}{ Outpatient care } \\
\hline All-cause visits (PPY) & 13.17 & 13.38 & 11.90 & 11.78 \\
\hline All-cause visits to a relevant specialist (PPY) & 2.77 & 2.74 & 2.24 & 2.41 \\
\hline Visits for NMO, ON, or TM (PPY) & 6.18 & 6.44 & 4.66 & 3.49 \\
\hline $\begin{array}{l}\text { Visits to a relevant specialist for NMO, } \\
\text { ON, or TM (PPY) }\end{array}$ & 1.83 & 1.96 & 0.91 & 1.27 \\
\hline
\end{tabular}

\section{Hospitalizations}

All-cause hospitalizations

Stays (PPY)

9.29

Days (PPY)

88.95

Hospitalizations with a primary or secondary diagnosis of NMO, TM, or ON

Stays (PPY)

Days (PPY)

All non-NMOSD-related hospitalizations

Stays (PPY)

Days (PPY)

Rehabilitation

All-cause rehabilitation

Stays (PPY)

Days (PPY)

Rehabilitation with a diagnosis

of NMO, TM, or ON

Stays (PPY)

Days (PPY)

Sick leave

All-cause sick leave

Sick leave periods (PPY)

Sick leave days (PPY)

NMO-related sick leave

Sick leave periods (PPY)

Sick leave days (PPY)

6.65

0.21

6.65
0.23

7.15

0.23

0.01

0.01

7.15

0.69

1.05

Analyses of sick leave and rehabilitation stays were based on AOK PLUS data only. Relevant specialists include neurologists, neuroradiologists, rheumatologists and ophthalmologists

$N M O$ neuromyelitis optica, $O N$ optic neuritis, $P P Y$ per patient year, $T M$ transverse myelitis

$\begin{array}{rrrr}1.29 & 1.38 & 0.57 & 0.70 \\ 49.11 & 52.84 & 12.89 & 18.57\end{array}$

$\begin{array}{rrrr}1.29 & 1.38 & 0.01 & 0.02 \\ 49.11 & 52.84 & 4.93 & 7.53\end{array}$


Table 3 Comparison of healthcare costs during active and inactive disease phases

\begin{tabular}{|c|c|c|c|c|}
\hline \multirow{2}{*}{$\begin{array}{l}\text { Healthcare costs [reported per patient month } \\
\text { (PPM) during the follow-up period] }\end{array}$} & \multicolumn{2}{|l|}{ Active } & \multicolumn{2}{|l|}{ Inactive } \\
\hline & Base cohort & Sensitivity cohort & Base cohort & Sensitivity cohort \\
\hline Total costs & $€ 7159.08$ & $€ 7632.38$ & $€ 714.17$ & $€ 834.91$ \\
\hline \multicolumn{5}{|l|}{ Hospitalization-related costs } \\
\hline All-cause hospitalizations (PPM) & $€ 6424.09$ & $€ 6881.87$ & $€ 259.10$ & $€ 267.11$ \\
\hline $\begin{array}{l}\text { Hospitalizations with a main or primary diagnosis } \\
\text { of NMO, TM, or ON (PPM) }\end{array}$ & $€ 5895.04$ & $€ 6315.38$ & $€ 46.78$ & $€ 67.55$ \\
\hline All non-NMOSD-related hospitalizations (PPM) & $€ 529.05$ & $€ 566.49$ & $€ 212.32$ & $€ 199.56$ \\
\hline \multicolumn{5}{|l|}{ Outpatient prescription costs } \\
\hline All treatment (PPM) & $€ 412.83$ & $€ 403.92$ & $€ 271.58$ & $€ 342.59$ \\
\hline Acute treatment $(\mathrm{PPM})$ & $€ 162.42$ & $€ 162.52$ & $€ 0.52$ & $€ 0.75$ \\
\hline Maintenance treatment (PPM) & $€ 46.43$ & $€ 49.18$ & $€ 22.11$ & $€ 31.65$ \\
\hline Other drugs (PPM) & $€ 0.00$ & $€ 0.00$ & $€ 5.27$ & $€ 0.00$ \\
\hline Pain medications (PPM) & $€ 63.17$ & $€ 63.43$ & $€ 38.13$ & $€ 36.71$ \\
\hline \multicolumn{5}{|l|}{ Cost of aids and remedies } \\
\hline All aids and remedies (PPM) & $€ 224.37$ & $€ 241.39$ & $€ 141.08$ & $€ 173.73$ \\
\hline \multicolumn{5}{|l|}{ Cost of rehabilitation } \\
\hline All-cause rehabilitation & $€ 97.78$ & $€ 105.20$ & $€ 42.40$ & $€ 51.48$ \\
\hline $\begin{array}{l}\text { Rehabilitation with a primary diagnosis of NMO, } \\
\text { TM, or ON }\end{array}$ & $€ 97.78$ & $€ 105.20$ & $€ 11.69$ & $€ 17.86$ \\
\hline
\end{tabular}

Analyses of costs related to aids and remedies, as well as rehabilitation stays, were based on AOK PLUS data only. Relevant specialists include neurologists, neuroradiologists, rheumatologists, and ophthalmologists

$N M O$ neuromyelitis optica, $N M O S D$ neuromyelitis optica spectrum disorder, $O N$ optic neuritis, $P P M$ per patient month, $T M$ transverse myelitis

during active and inactive periods PPM for both the base cohort of NMOSD patients, as well as the sensitivity cohort.

\section{HCRU and Cost Comparison Between Patients with and without NMOSD}

In our PSM analysis of HCRU and cost in patients with and without NMOSD, matching strategies of 1:16 (AOK PLUS dataset) and 1:13 (GWQ dataset) were found to lead to the best possible result (i.e., minimization of the average percentage bias between groups). All identified patients with NMOSD $(N=130)$ were included and matched with those from the base cohort of patients without NMOSD $(N=5,402,103)$, resulting in a total matched population of 130 patients with NMOSD, and 1822 patients without NMOSD.

Supplementary Table 2 provides an overview of the baseline characteristics of patients included in both cohorts before and after matching, for both AOK PLUS and GWQ datasets. During the matching process, all statistically significant differences $(P<0.001)$ between the two comparison groups in terms of sex, comorbidities, and baseline HCRU were eliminated. This means that non-NMOSD patients included in our comparison were approximately as comorbid and used approximately as many healthcare 
Table 4 Comparing HCRU among patients with and without NMOSD

\begin{tabular}{lccc}
\hline $\begin{array}{l}\text { Healthcare resource utilization } \\
\text { (measured during the follow-up period) }\end{array}$ & NMOSD cohort & Non-NMOSD cohort & $P$-value \\
\hline Outpatient care & 12.05 & 10.00 & $<0.001$ \\
All-cause visits (PPY) & 7.57 & 6.19 & $<0.001$ \\
Outpatient specialist visits (PPY) & 4.48 & 3.81 & $<0.001$ \\
Outpatient GP visits (PPY) & & & $<0.001$ \\
Hospitalizations & 0.92 & 0.49 & $<0.001$ \\
All-cause hospitalizations (PPY) & 9.83 & 4.17 & \\
All-cause hospitalization days (PPY) & & & $<.135$ \\
Rehabilitation & 0.06 & 0.04 & $<0.001$ \\
All-cause rehabilitation stays (PPY) & 2.48 & 0.84 & \\
All-cause rehabilitation days (PPY) & & & $<.012$ \\
Sick leave & 0.59 & 0.75 & $<0.001$ \\
All-cause sick leave periods (PPY) & 13.72 & 8.23 & \\
All-cause sick leave days (PPY) &
\end{tabular}

Analyses of sick leave and rehabilitation stays were based on AOK PLUS data only

GP general practitioner, NMOSD neuromyelitis optica spectrum disorder; $P P Y$ per patient year

resources during the baseline period, as those with NMOSD.

All HCRU outcomes and associated costs were higher for NMOSD patients than their non-NMOSD counterparts. Patients with NMOSD recorded 12.05 outpatient visits PPY compared to 10.00 PPY in the non-NMOSD cohort. The number of hospitalization days was twice as high among NMOSD patients (9.83/ 4.17 PPY), and similar trends were observed for sick leave days (13.72/8.23 days PPY). The results of the full HCRU and cost comparison are outlined in Tables 4 and 5 .

The difference in total healthcare costs PPY was $€ 8245.62$, with almost triple the cost in NMOSD patients (€12,913.28 PPY) as compared to patients without NMOSD ( $€ 4667.66 \mathrm{PPY}$ ). Hospitalizations (€6448.32/€1937.64 PPY) and outpatient prescriptions (€3335.67/€1037.64 PPY) contributed most strongly to the difference; however, differences in spending on rehabilitation (€527.39/€120.14), aids and remedies (€1720.89/€740.63), and outpatient care (€881.01/€831.60) were also observed.
Given the small sample size and the potential bias introduced by the inclusion of a few patients with outlier healthcare costs (including one patient with repeated prescriptions for an orphan drug in the base NMOSD cohort), a sensitivity cost analysis was conducted entailing the exclusion of the top and bottom $2.5 \%$ of patients in terms of total healthcare costs PPY (based on outpatient prescriptions, outpatient care and hospitalization costs only) from each of the matched cohorts. This resulted in a decrease in the associated costs of outpatient prescriptions PPY across both groups ( $€ 1455.31 /$ $€ 891.28)$. Table 5 shows the results of both analyses.

\section{DISCUSSION}

Our study confirms findings from the USA that patients with NMOSD incur substantially higher healthcare costs than matched patients without NMOSD, and that the economic and 
Table 5 Comparing healthcare costs among patients with and without NMOSD

\begin{tabular}{llll}
\hline Healthcare Costs (reported PPY during the follow-up period) & NMOSD cohort & Non-NMOSD cohort & $\boldsymbol{P}$-value \\
\hline Overall results & & & \\
Total costs (PPY) & $€ 12,913.28$ & $€ 4667.66$ & $<0.001$ \\
Hospitalizations (PPY) & $€ 6448.32$ & $€ 1937.64$ & $<0.001$ \\
Outpatient care (PPY) & $€ 881.01$ & $€ 831.60$ & $<0.001$ \\
Outpatient prescriptions (PPY) & $€ 3335.67$ & $€ 1037.64$ & $<0.001$ \\
Rehabilitation (PPY) & $€ 527.39$ & $€ 120.14$ & $<0.001$ \\
Aids and remedies (PPY) & $€ 1720.89$ & $€ 740.63$ & $<0.001$ \\
Sensitivity cost analysis & & & $<0.001$ \\
Total costs (PPY) & $€ 10,520.94$ & $€ 4135.14$ & $<0.001$ \\
Hospitalizations (PPY) & $€ 6089.78$ & $€ 1608.22$ & $<0.001$ \\
Outpatient care (PPY) & $€ 903.68$ & $€ 821.17$ & $<0.001$ \\
Outpatient prescriptions (PPY) & $€ 1455.31$ & $€ 891.28$ & $<0.001$ \\
Rehabilitation (PPY) & $€ 533.56$ & $€ 111.48$ & $<0.001$ \\
Aids and remedies (PPY) & $€ 1538.61$ & $€ 702.99$ & \\
\hline
\end{tabular}

Analyses of costs related to aids and remedies, as well as rehabilitation stays, were based on AOK PLUS data only NMOSD neuromyelitis optica spectrum disorder; PPY per patient year

health resource burden is the greatest during periods of active disease $[16,17]$.

Among a population of 9 million statutory insured patients in Germany, we identified 130 patients with NMOSD using our base algorithm and 98 patients using a sensitivity approach. This corresponds to a prevalence rate of around $1.44 / 100,000$ patients, which is well aligned with the overall prevalence figures from the literature (0.5-4 cases/100,000 patients) [2, 3]. Our slightly more conservative detection technique used in the sensitivity analysis yielded a lower prevalence of 1.09/100,000, with higher comparability to prevalence figures found in other northern European nations, including Denmark (1.09/100,000) and Sweden (1.04/ $100,000)[21,22]$. The average age of patients included in our study (base cohort/sensitivity: 46.8/46.6 years) was comparable to those observed in two US claims data studies, with respective averages of 44.9 and 46 years $[16,17]$. However, the percentage of females was marginally lower (base cohort/sensitivity: 58\%/ $64 \%)$ than figures cited elsewhere in the literature $(66-88 \%)[16,17,23]$.

According to NEMOS, an open access network which operates a registry for NMOSD patients in Germany, the most common first line maintenance therapies for treating NMOSD include off-label rituximab and azathioprine [13]. Ultimately, $18 \%$ and $10 \%$ of patients in the base cohort were prescribed rituximab and azathioprine, respectively, within 1 year of their index $\mathrm{NMO} / \mathrm{TM} / \mathrm{ON}$ diagnosis. In addition, use of mycophenolate mofetil was recorded at lower levels $(2 \%)$ within the NMOSD patient population. While utilization of other second-line and third-line agents including mitoxantrone, methotrexate and tocilizumab was not detected, this may be due to the follow-up period of one year used to measure treatment.

With regard to disease activity, a higher percentage of patients in our study (base cohort/sensitivity: $53.1 \% / 66.3 \%)$ experienced 
at least one relapse, when compared to findings from two US claims data studies, which reported respective rates of $41.2 \%$ and $47.7 \%[16,17]$. Annualized rates of NMOSD relapses derived in this study ranged from 0.52 to 0.66 . This is slightly less than figures from the USA (ARR = 0.8 ) and one retrospective case control study from Australia and New Zealand $(A R R=0.77)$ $[10,17]$. Nevertheless, rates achieved in this study are reasonable, given that patients with NMOSD have been found to experience both clustered and non-clustered relapses, and utilization of different maintenance therapy options may play a role in the frequency of relapses [24]. Lastly, one multi-center study from the USA found that consistent use of azathioprine, mycophenolate mofetil, and rituximab may lower the ARR to 0.14-0.63 [25].

In terms of cost, a recent study from Japan concluded that physical impairment and permanent disability in NMOSD is largely linked to periods of acute relapses [15], or active disease periods, as we have referred to them in this research. Our study reinforces these findings since healthcare resources and costs were found to be disproportionately consumed and incurred during periods of active disease phases, with a resultant cost ratio of $€ 7159.08 /$ active month versus $€ 714.17 /$ inactive month of observation. This notion was further supported by another claims data study from the USA which reported that annualized overall costs were four times as high in patients who relapsed than in patients who did not experience a clinical relapse. The authors concluded that hospitalizations served as the primary driver of differential health spending, corresponding with an average cost of $\$ 44,137$ in patients with at least one relapse versus $\$ 1121$ in patients without a relapse.

Within our matched comparison, we find a threefold difference in annualized direct healthcare spending, corresponding with $€ 12,913.28$ PPY in patients with NMOSD vs. $€ 4667.66$ PPY in patients without NMOSD. This finding is similar to the results achieved in another annualized cost comparison between patients with and without MS in Germany from 2013, which produced similar estimates of $€ 14,240$ PPY vs. €4214 PPY in MS vs. non-MS patients [26]. Moreover, a recent claims data study from the USA measured differences in annualized spending among patients with NMOSD and a comparison group, using a similar strategy for identifying NMOSD patients and clinical relapses (based on primary inpatient diagnoses and codes for acute treatments including methylprednisolone, IVIG and PLEX) [17]. The magnitude of the difference in total healthcare costs between patients with and without NMOSD was about twice as large in this study ( $\$ 60,599$ versus $\$ 8912$, around 7:1) as in our own $(€ 12,913.28$ versus $€ 4667.66$, around $3: 1)$. This likely reflects general differences in the annualized per-patient healthcare costs in Germany and the USA [27]. Most importantly, the authors also concluded that differences in total healthcare costs between patients with and without NMOSD were foremost attributable to more hospital admissions and longer hospital stays, as well as higher spending on outpatient prescriptions.

\section{Limitations}

This analysis has several limitations common to claims data studies. In Germany, insurance claims are developed for billing purposes, thus data on certain diagnostic screening measures (including serology testing for AQP4-IgG antibodies) and results from brain and spinal cord MRIs were not available. However, in line with findings from studies on algorithm validation, the inclusion of AQP4-IgG serology testing in the patient finding algorithm may have helped to increase the specificity of the patient detection algorithm [20]. Nevertheless, a lack of access to relevant clinical data on biomarker test results and some screening measures represents one key limitation of our claims-driven approach, also because some myelin oligodendrocyte glycoprotein (MOG)-IgG-seropositive patients might fulfill the 2015 NMOSD diagnostic criteria and might be accidentally included in this claims analysis, although myelin oligodendrocyte glycoprotein antibody disease (MOGAD) is now perceived as a distinct disease entity from AQP4 + NMOSD. Since information on key biomarkers and clinical data was not accessible, identification of patients with 
NMOSD was performed exclusively based on ICD-10 codes. ICD-10 codes have not yet been fully validated for use in identifying NMOSD patients in claims data, and authors from a linked data study recently concluded that the application of the neuromyelitis optica ICD-9 code alone is generally inaccurate for the identification of NMOSD in claims research [20]. To increase the positive predictive value of our ICD-10 based case-identifying algorithm, two identification strategies were developed and tested, incorporating multiple NMO and symptom codes, as well as MS and sarcoidosis exclusion criteria. While the patient detection algorithm used to identify patients with NMOSD was developed based on a rigorous review of claims data analyses and algorithm validation studies, our chosen algorithm has not undergone a formal validation process. As a result, the exact specificity and sensitivity of the algorithm cannot be directly observed.

In our study, no differentiation is made between HCRU during periods of relapse and pseudo-relapse. This is largely due to the implausibility of differentiating between relapse and pseudo-relapse within a study using only claims data-since no data on MRI outcomes or inpatient treatment with intravenous corticosteroids was available. Ultimately, we opted to consider all hospitalizations with a main diagnosis of NMO, TM, or ON as "active" periods, so as not to underreport relapse figures given the limited availability of some key data points.

Within the existing body of literature, more emphasis has been placed upon characterization of annualized relapses rates and periods between attacks, than on the corresponding duration of NMOSD relapses. Thus, another limitation derives from the fact that there is limited data on the length of relapses in patients with NMOSD. In the German context, this study is the first claims data analysis of NMOSD. Our approach for detecting relapses among NMOSD patients in German claims data draws on the International Panel for NMO Diagnosis' (IPND) revised criteria for diagnosing NMOSD, as well as findings from a retrospective case control study which identified a median relapse duration of 30 days in patients with NMOSD [10]. While our use of a 30-day period for tracking disease was further reinforced by methods applied in similar studies [17], we acknowledge that the use of a standard window constitutes one weakness of the applied methodology, given possible heterogeneity in relapse duration which may lead to the misclassification of outcomes.

The decision to classify all inpatient hospitalizations with a main diagnosis of NMO/TM/ $\mathrm{ON}$ as periods of active disease was made to account for the fact that NMOSD attacks are often treated in an inpatient setting. This decision may have introduced some bias to the HCRU and cost analysis since inpatient costs are generally more substantial than other healthcare costs. To address this potential limitation, we reported the difference in the number of non-NMOSD-related hospitalizations, and their associated costs to allow for more robust conclusions.

HCRU and cost was analyzed during periods of active and inactive disease. In our attribution of costs to active and inactive periods, spending on inpatient care was allocated equally across all hospitalization and rehabilitation days. However, while this strategy was used to attribute aggregate costs to both periods of inactive and active disease, we acknowledge that the costs of important testing, imaging, and other procedures are not incurred equitably across all hospitalization/rehabilitation days, and thus this assumption may not be well-aligned with a real-world setting. Ultimately, averaging costs over an entire inpatient period and proportionately allocating them to active and inactive phases proved to be the most logical strategy for comparing costs across active and inactive disease periods, given the data limitations.

Lastly, this study only captures direct measurable healthcare costs and likely underestimates real health and social care costs as well as the societal and emotional burden on patients and their families.

\section{CONCLUSION}

The health economic burden posed by NMOSD in Germany is substantial. Patients with NMOSD consume substantially more healthcare 
resources and incur higher direct healthcare costs during active disease phases than during inactive periods. This primarily results from the need for acute treatment which is often provided in inpatient settings. Therefore, the prevention of relapses with highly efficacious maintenance treatment options represents one strategy to minimize direct high costs incurred by patients with NMOSD as well as the overall disease burden.

\section{ACKNOWLEDGEMENTS}

Funding. The study and Rapid Service Fee was financed by F. Hoffmann-La Roche Ltd.

Authorship . All named authors meet the International Committee of Medical Journal Editors (ICMJE) criteria for authorship for this article, take responsibility for the integrity of the work as a whole, and have given their approval for this version to be published.

Authors' Contributions. Conceptualization, RK, FH, TW, JM, SS; methodology, RK, FH, TW, JM, SS, UM, BD; formal analysis, RK, FH; writing-original draft preparation, $\mathrm{RK}, \mathrm{FH}$; writing - review and editing, RK, FH, TW, JM, SS, UM, $\mathrm{BD}$; All authors have read and agreed to the final version of the manuscript.

Prior Presentation. Some results from this study were summarized in two research abstracts and conference posters, presented at the ECTRIMS 2021 Virtual Congress (13-15 October 2021).

Disclosures. Rachel Knapp and Fränce Hardtstock are employees of Cytel Inc. Thomas Wilke participated in this study as an employee of IPAM e.V. Barthold Deiters is an employee of GWQ ServicePlus AG, one of the data providers for this study. Ulf Maywald works for one of the insurance funds (AOK PLUS) that provided the data. Julie Mouchet and Sophie Schneider are full-time permanent employees of F. Hoffmann-La Roche Ltd.
Compliance with Ethical Guidelines. This study involved the use of two anonymized health insurance claims datasets provided by AOK PLUS and GWQ ServicePLUS under formal agreement and legal basis of $\$ 75$, Tenth Book of the Social Code (SGB X). Accordingly, no informed consent or ethical approval from an institutional review board was required to implement this retrospective study, which only used anonymized, non-identifiable data.

Data Availability. The datasets generated during and/or analyzed during the current study are not publicly available since the findings of this study are extracted from individual patient records. Data were available for research purposes from the sickness fund upon request, in an anonymized form. Due to restrictions around revealing patients' confidential information, data were used under license for the current study, and so are neither publicly available nor can be shared further.

Open Access. This article is licensed under a Creative Commons Attribution-NonCommercial 4.0 International License, which permits any non-commercial use, sharing, adaptation, distribution and reproduction in any medium or format, as long as you give appropriate credit to the original author(s) and the source, provide a link to the Creative Commons licence, and indicate if changes were made. The images or other third party material in this article are included in the article's Creative Commons licence, unless indicated otherwise in a credit line to the material. If material is not included in the article's Creative Commons licence and your intended use is not permitted by statutory regulation or exceeds the permitted use, you will need to obtain permission directly from the copyright holder. To view a copy of this licence, visit http://creativecommons.org/licenses/by$\mathrm{nc} / 4.0 /$.

\section{REFERENCES}

1. Lana-Peixoto MA, Talim N. Neuromyelitis optica spectrum disorder and anti-MOG syndromes 
[Internet]. Vol. 7, Biomedicines. MDPI AG; 2019 [cited 2021 Jun 3]. hhttps://www.ncbi.nlm.nih.gov/ pmc/articles/PMC6631227/. Accessed 3 June 2021.

2. Hor JY, Asgari N, Nakashima I, Broadley SA, Leite MI, Kissani N, et al. Epidemiology of neuromyelitis optica spectrum disorder and its prevalence and incidence worldwide [Internet]. Vol. 11, Frontiers in neurology. Frontiers Media S.A.; 2020 [cited 2021 Jun 3]. p. 501. https://www.frontiersin.org. . Accessed 3 June 2021.

3. Jarius S, Wildemann B, Paul F. Neuromyelitis optica: Clinical features, immunopathogenesis and treatment. Clin Exp Immunol [Internet]. 2014 May [cited 2021 Jun 3];176(2):149-64. https://www. ncbi.nlm.nih.gov/pmc/articles/PMC3992027/. Accessed 3 June 2021.

4. Mealy MA, Wingerchuk DM, Greenberg BM, Levy M. Epidemiology of neuromyelitis optica in the United States: a multicenter analysis. Arch Neurol [Internet]. 2012 Sep 1 [cited 2021 Jun 3];69(9): 1176-80. https://jamanetwork.com. Accessed 3 June 2021.

5. Jarius S, Ruprecht K, Wildemann B, Kuempfel T, Ringelstein M, Geis C, et al. Contrasting disease patterns in seropositive and seronegative neuromyelitis optica: a multicentre study of 175 patients. J Neuroinflammation [Internet]. 2012 Jan 19 [cited 2021 Jun 3];9(1):1-17. http://www. jneuroinflammation.com/content/9/1/14. Accessed 3 June 2021.

6. Wingerchuk DM, Banwell B, Bennett JL, Cabre P, Carroll W, Chitnis T, et al. International consensus diagnostic criteria for neuromyelitis optica spectrum disorders [Internet]. Vol. 85, Neurology. Lippincott Williams and Wilkins; 2015 [cited 2021 Jun 3]. p. 177-89. https://n.neurology.org/content/85/ 2/177. Accessed 3 June 2021.

7. Kessler RA, Mealy MA, Levy M. Early indicators of relapses vs pseudorelapses in neuromyelitis optica spectrum disorder. Neurol Neuroimmunol Neuroinflamm. 2016;3(5):e269.

8. Banerjee A, Ng J, Coleman J, Ospina JP, Mealy M, Levy M. Outcomes from acute attacks of neuromyelitis optica spectrum disorder correlate with severity of attack, age and delay to treatment. Mult Scler Relat Disord [Internet]. 2019 Feb 1 [cited 2021 Jun 3];28:60-3. https://www.ncbi.nlm.nih.gov/ pmc/articles/PMC6397696/. Accessed 3 June 2021.

9. Mealy MA, Boscoe A, Caro J, Levy M. Assessment of patients with neuromyelitis optica spectrum disorder using the EQ-5D. Int J MS Care [Internet]. 2019 May 1 [cited 2021 Jun 3];21(3):129-34. https:// www.ncbi.nlm.nih.gov/pmc/articles/ PMC6397696/. Accessed 3 June 2021.
10. Khalilidehkordi E, Clarke L, Arnett S, Bukhari W, Jimenez Sanchez S, O'Gorman C, et al. Relapse patterns in NMOSD: evidence for earlier occurrence of optic neuritis and possible seasonal variation. Front Neurol [Internet]. 2020 Jun 16 [cited 2021 Jun 3];11:537. www.frontiersin.org. Accessed 3 June 2021.

11. Masuda H, Mori M, Uzawa A, Muto M, Uchida T, Ohtani R, et al. Recovery from optic neuritis attack in neuromyelitis optica spectrum disorder and multiple sclerosis. J Neurol Sci [Internet]. 2016 Aug 15 [cited 2021 Jun 3];367:375-9. https://pubmed. ncbi.nlm.nih.gov/27423624/. Accessed 3 June 2021.

12. Kessler RA, Mealy MA, Levy M. Treatment of neuromyelitis optica spectrum disorder: acute, preventive, and symptomatic [Internet]. Vol. 18, Current treatment options in neurology. Current Science Inc.; 2016 [cited 2021 Jun 3]. p. 1-15. https:// pubmed.ncbi.nlm.nih.gov/26705758/. Accessed 10 Sept 2021.

13. Trebst C, Jarius S, Berthele A, Paul F, Schippling S, Wildemann $\mathrm{B}$, et al. Update on the diagnosis and treatment of neuromyelitis optica: recommendations of the Neuromyelitis Optica Study Group (NEMOS) [Internet]. Vol. 261, J Neurol. Springer; 2014 [cited 2021 Jun 22]. p. 1-16. http://www. nemos-net.de. Accessed 10 Sept 2021.

14. Biswas, Mukherjee A. Therapy of NMO spectrum disorders. Ann Indian Acad Neurol [Internet]. 2015 Sep 1 [cited 2021 Sep 10];18(5):16. https://www. annalsofian.org/article. asp?issn=0972-2327; year= 2015; volume $=18$;issue $=5$; spage $=16$; epage $=23$; aulast =Biswas. Accessed 10 Sept 2021.

15. Akaishi T, Takahashi T, Misu T, Abe M, Ishii T, Fujimori J, et al. Progressive patterns of neurological disability in multiple sclerosis and neuromyelitis optica spectrum disorders. Sci Rep [Internet]. 2020 Dec 1 [cited 2021 Jun 3];10(1):13890. https://www. nature.com/articles/s41598-020-70919-w. Accessed 3 June 2021.

16. Stafkey-Mailey D, Boscoe A, Ajmera M, Mauskopf J, Iloeje U, Levy M. A Real-world analysis of relapses and costs of neuromyelitis optica spectrum disorders using a United States Administrative Claims Database (P6.161). Neurology. 2016;86(16 Supplement):P6.161. https://n.neurology.org/content/86/ 16_Supplement/P6.161/tab-article-info. Accessed 3 June 2021.

17. Royston M, Kielhorn A, Weycker D, Shaff M, Houde L, Tanvir I, et al. Neuromyelitis optica spectrum disorder: clinical burden and cost of relapses and disease-related care in US Clinical Practice. Neurol Ther [Internet]. 2021. https://doi.org/10.1007/ s40120-021-00253-4 (cited 2021 Jun 3). 
18. Ajmera MR, Boscoe A, Mauskopf J, Candrilli SD, Levy M. Evaluation of comorbidities and health care resource use among patients with highly active neuromyelitis optica. J Neurol Sci [Internet]. 2018 Jan 15 [cited 2021 Jun 3];384:96-103. https:// pubmed.ncbi.nlm.nih.gov/29249387/. Accessed 3 June 2021.

19. Lee HL, Kim JY, Seok JM, Hong YH, Lim NG, Shin $\mathrm{HY}$, et al. Prevalence and incidence of neuromyelitis optica spectrum disorder in Korea: population based study. J Korean Med Sci [Internet]. 2020 May 1 [cited 2021 Jun 3];35(17). http://www.ncbi.nlm. nih.gov/pmc/articles/PMC7200182/. Accessed 3 June 2021.

20. Gmuca S, Hardy DI, Narula S, Stoll S, Harris J, Zhao $Y$, et al. Validation of claims-based diagnoses of adult and pediatric neuromyelitis optica spectrum disorder and variations in diagnostic evaluation and treatment initiation. Mult Scler Relat Disord. 2020;37:101488.

21. Jonsson DI, Sveinsson O, Hakim R, Brundin L. Epidemiology of NMOSD in Sweden from 1987 to 2013: A nationwide population-based study. Neurology [Internet]. 2019 Jul 9 [cited 2021 Jun 18];93(2):E181-9. https://www.ncbi.nlm.nih.gov/ pmc/articles/PMC6656652/. Accessed 18 June 2021.

22. Papp V, Illes Z, Magyari M, Koch-Henriksen N, Kant $M$, Pfleger CC, et al. Nationwide prevalence and incidence study of neuromyelitis optica spectrum disorder in Denmark. Neurology [Internet]. 2018 [cited 2021 Jun 18];91(24):E2265-75. https://www. ncbi.nlm.nih.gov/pmc/articles/PMC6329324/.

Accessed 18 June 2021.
23. Pandit L, Asgari N, Apiwattanakul M, Palace J, Paul F, Leite MI, et al. Demographic and clinical features of neuromyelitis optica: a review [Internet]. Vol. 21, Multiple Scler J. SAGE Publications Ltd; 2015 [cited 2021 Jun 18]. p. 845-53. https://www.ncbi.nlm. nih.gov/pmc/articles/PMC4463026/. Accessed 18 June 2021.

24. Akaishi T, Nakashima I, Takahashi T, Abe M, Ishii T, Aoki M. Neuromyelitis optica spectrum disorders with unevenly clustered attack occurrence. Neurol Neuroimmunol Neuroinflamm [Internet]. 2020 Jan 1 [cited 2021 Jun 22];7(1):640. https://nn. neurology.org/content/7/1/e640. Accessed 22 June 2021.

25. Mealy MA, Wingerchuk DM, Palace J, Greenberg BM, Levy M. Comparison of relapse and treatment failure rates among patients with neuromyelitis optica: Multicenter study of treatment efficacy. JAMA Neurol [Internet]. 2014 Mar 1 [cited 2021 Jun 22];71(3):324-30. https://jamanetwork.com/. Accessed 22 June 2021.

26. König C, Altevers J, Maas C, Meise D, Bierbaum M, Riederer $\mathrm{C}$, et al. Burden of multiple sclerosis in Germany-a matched cohort study using a large claims database. ISPOR Eur [Internet]. 2019 [cited 2021 Nov 2]; http://www.bbsr.bund.de. Accessed 2 Nov 2021.

27. Health spending, Total, US dollars/capita, 2017 [Internet]. [cited 2021 Jun 22]. https://data.oecd. org/chart/5F2L. Accessed 2 Nov 2021. 